\title{
Psychiatric disorder, stress and burn-out
}

\author{
E. Guthrie \& D. Black
}

This paper will focus primarily on the most recent research studies involving doctors in the UK. We will not discuss the treatment of doctors who are psychiatrically unwell as this is covered in other papers elsewhere in this issue. Before reviewing specific studies, we will consider the measurement of psychiatric disorder, stress and burn-out.

\section{Measurement and definitions}

\section{Psychiatric disorder}

Studies of psychiatric disorder in doctors have mainly been confined to the study of minor psychiatric morbidity. Other indicators of more severe disorder, such as suicide, have been used, but not as frequently. Most UK studies have used Goldberg's General Health Questionnaire to screen for psychological morbidity. Different forms of the questionnaire and different cut-off points have been used, which can make comparisons difficult. The GHQ-12 (Goldberg \& Williams, 1988) has been most widely employed with a conservative cutoff of three-quarters. Borrill et al (1996) have recently validated the GHQ-12 in a large sample of health service employees using the Clinical Interview Schedule. They found that threequarters was the best cut-off score yielding an estimated sensitivity of $73 \%$ and specificity of $86 \%$. These validity coefficients match those reported in other studies on the GHQ and are acceptable.

There are very few studies in doctors in which a standardised semi-structured interview has been used to assess psychiatric disorder. One such study is that by Wrate \& Baldwin in Edinburgh (see pp.
290-296, this issue) who are following-up a group of approximately 200 junior doctors, who were assessed in detail as medical students. The study is still in progress. When completed, it will be the most detailed study of junior doctors that has ever been undertaken.

\section{Burn-out}

Burn-out is a constellation of symptoms which develop in professionals in relation to work. Previously hard-working, diligent and committed individuals become increasingly dissatisfied, disillusioned and cynical in relation to work and other aspects of their lives. Doctors who previously cared about their patients find themselves dreading clinical work and becoming more and more detached and uncaring towards patients. Pines et al (1981), highlighted the important depressive component of the condition and referred to:

physical depletion, feelings of helplessness and hopelessness, emotional drain, the development of negative attitudes to work, life and other people ... a sense of distress and failure in the quest for ideals.

Freudenburger \& Richelson (1980) highlighted the sense of the loss of idealism in a previously energised and committed individual. They described it as:

a state of fatigue or frustration brought about by devotion to a cause, way of life, or relationship that failed to produce the expected reward.

Edelwich \& Brodsky (1980) defined burn-out as:

a progressive loss of idealism, energy, purpose, and concern as a result of conditions of work.

\footnotetext{
E. Guthrie is a senior lecturer in liaison psychiatry at the University of Manchester, School of Psychiatry and Behavioural Sciences, Rawnsley Building, Manchester Royal Infirmary, Oxford Road, Manchester M13 9WL, interested in psychotherapy and the nature of stress and psychological morbidity in medical students. D. Black is a consultant psychiatrist at Hope Hospital, Salford. Dr Black has been involved in a five-year project examining the prevalence of psychological morbidity in medical students. She is also interested in research into the effects of day hospital and community treatment for patients with psychiatric disorder.
} 
It can be argued that doctors are particularly susceptible to burn-out because of the high ideals of the profession and the long working hours. The health service reforms have also placed additional pressures upon doctors, increasing their workload, decreasing a sense of support, increasing patient expectation, and greater support for patients wishing to make complaints against doctors.

The two most widely used measures of burnout are the Maslach Burn-Out Inventory (MBI; Maslach \& Jackson, 1986) and the Burn-Out Measure (Pines et al, 1981). The Burn-Out Measure has not been used on a British population of doctors. It was, however, used recently in the USA in a sample of 440 middle-aged physicians who had previously been screened with a personality trait measure (Minnesota Multiphasic Personality Inventory (MMPI); Graham, 1987) 25 years earlier (McCranie \& Brandsma, 1988). Not surprisingly, given the large time difference, little association was found between the scores on the MMPI when the physicians were young men and the burn-out ratings when they were middle-aged professionals.

The MBI has been used in several studies of health professionals, including doctors, although it has been used in only one large-scale study of UK doctors (Ramirez et al, 1996). The MBI has three subscales: emotional exhaustion, depersonalisation (feelings of detachment and callousness towards people at work) and personal accomplishment (feelings of competency and achievement at work). The total score for each subscale is categorised 'low', 'average' or 'high' according to predetermined cut-off scores based on normative data from a sample of American health professionals. A high degree of burn-out is indicated by high scores on the emotional exhaustion and depersonalisation subscales and low scores on the personal accomplishment subscale.

There is considerable overlap between the emotional exhaustion subscale of the MBI and the symptomatology of depression. Glass et al (1996) conducted multiple regression analyses on each of the MBI subscales using the Beck Depression Inventory as one of the predictor variables in a sample of 162 nurses. They found that over $19 \%$ of the variance in the emotional energy subscale was attributable to the scores on the Beck Depression Inventory, whereas the percentages of the variance on the depersonalisation and personal accomplishment subscales were only 3.9 and $6.5 \%$, respectively.

Thus, burn-out is not just an alternative way of viewing depression. Although the emotional exhaustion subscale and depressive symptomalogy share appreciable variance, the other two subscales appear to measure different entities.

\section{Stress}

Stress is an ill-defined term and its use has been heavily criticised. The word 'stress' is derived from the Latin word stringere which means 'to draw tight'. Most theories of stress involve two factors: an interaction between the individual and the environment, which results in a demand, and a failure of the individual to tolerate or integrate the demand. Most studies of stress in doctors have focused upon the first part of the concept; studying which particular aspects of a job create the most stress (British Medical Association, 1992). There are few well-recognised measures of stress and many studies have developed their own rating scales in which respondents are asked to rate various aspects of work in terms of how stressful the respondents perceive them to be. It is difficult to make comparisons between studies when different measures have been used.

A recognised measure of stress that has been used in UK studies of doctors is the Occupational Stress Indicator (Cooper et al, 1988). This is divided into six component scales, each of which is divided into a set of subscales. The scales are: sources of pressure in the job, coping skills, type A behaviour pattern, job satisfaction, locus of control and mental and physical ill-health.

There are some concerns regarding the psychological subscales of this questionnaire. Since this measure was developed, interest in issues to do with locus of control have waned. The mental health subscale is not a well-recognised measure of psychological distress, but it has been validated against the Crown-Crisp Experiential Index (1979).

Firth (1986) has also developed a checklist for measuring aspects of stress, particularly in relation to doctors. This measure was used in her study of junior hospital doctors and respondents are asked to rate on a four-point scale various aspects of medical-related work.

\section{Morbidity in doctors}

Although doctors have lower rates of many kinds of physical illness than the general population, past studies of sickness in the medical profession have highlighted three particular areas of concern. Doctors have higher rates of suicide, psychiatric illness and possibly alcohol and drug misuse than the general population.

The rate of suicide in doctors is three times that in the general population, but most of the increased rate is accounted for by women doctors (British 
Medical Association, 1993). In 1968 women doctors were almost four times more likely to kill themselves than the general population. Figures reported by Kelly et al (1995) for the period 19821992 are very similar, with women doctors being 3.22 times more likely to kill themselves than the general population. Male doctors are 1.4 times more likely to take their own lives (Box 1).

There is some evidence that the suicide rates for doctors peak in mid-life rather than showing a linear increase with age as for the general population (Steppacher \& Mauser, 1974). There is also evidence that suicide in doctors is underreported, as many deaths are not classified as suicide but as 'accidental poisoning by drugs' (Richings et al, 1986).

The high suicide rate in women doctors is of great concern and is difficult to explain, but one important factor is likely to be the higher prevalence of psychiatric morbidity in women doctors than their male counterparts. Studies have also shown that three-quarters of doctors who kill themselves are depressed at the time.

When students enter medical school the prevalence of psychiatric disorder is slightly, but not significantly, higher for women than men (Miller \& Surtees, 1991; Guthrie et al, 1995). As doctors progress in their careers, psychological morbidity increases disproportionately for women.

The recent large study by Borrill et al (1996) of the mental health of 12000 National Health Service (NHS) employees (including doctors) found the overall prevalence of psychological morbidity in doctors was $27 \%$. This was using the GHQ-12 and a conservative cut-off score of three-quarters, which had been validated on a subsample of 600 respondents. The prevalence of psychiatric illness for women doctors was $36 \%$, which was significantly higher than that for men (24\%).

Doctors are not the most psychologically disturbed occupational group in the NHS. Overall, health service managers and nurses reported higher rates of psychological morbidity than doctors in Borrill et al's (1996) study. All three groups, however, had significantly higher rates of psychological morbidity than employed workers (data from the British Household Panel Survey (BHPS), 3rd wave data, 1993-1994).

When the occupational groups in the NHS study were compared separately for each gender, female doctors and female managers were found to have very high rates of psychological morbidity compared with men in equivalent posts. For men, there was little variation in psychological morbidity across occupations, whereas for women, the prevalence rate varied from $17 \%$ for ancillary workers to $36 \%$ for doctors and $41 \%$ for managers.

\section{Box 1. Suicide risk and psychological morbidity in doctors compared with the general population}

\section{Suicide risk \\ Women doctors are 3.2 times more likely to commit suicide \\ Men doctors are 1.4 times more likely to commit suicide}

\section{Psychological morbidity \\ Overall prevalence in doctors is $\sim 27 \%$ \\ Prevalence in women doctors is $36 \%$ \\ Prevalence in men doctors is $24 \%$}

The higher levels of mental ill-health recorded by women managers and doctors compared with women in other NHS occupations were strongly associated with higher levels of perceived work demands, long working hours, working extra unpaid hours and the amount of face-to-face patient contact (doctors only). Despite these findings, the researchers found it difficult to explain the difference between men and women doctors, as all the above factors are equally relevant for both genders.

Rates of psychological morbidity for men and women are only slightly different until the age when women marry and have children. The rates for women then become much higher. Marriage is a protective factor for men in terms of psychiatric illness and a risk factor for women.

It is possible that trying to combine a professional career, which is exceptionally demanding in terms of long hours and additional commitments, with a traditional marriage and children, is particularly noxious for women. It is still the rule rather than the exception that in a marriage between two professionals, the woman, in addition to her job, takes on a greater share of the household commitments. It is also still the case that many male doctors are supported through their training by wives who play a subordinate role, whereas few female doctors have the luxury of house-husbands to support them.

This, however, does not explain the high rates of suicide in female doctors as the Office of Population Censuses and Surveys occupational mortality data are based on the rates reported for single women. This high suicide rate in female doctors warrants more detailed research. In particular, we need to understand the complex interplay between work demands and other 
psychosocial factors, and we need better ways of identifying doctors who are at risk of suicide.

Although doctors as a profession have drastically reduced the amount that they smoke, there is little evidence of a reduction in alcohol consumption. Although some studies have suggested that doctors may have higher than expected rates of alcoholism (Murray, 1976; Brooke et al, 1991) the evidence is equivocal, and Clare, who reviewed this subject in 1990, concluded that there was little evidence to suggest that doctors drink more than other professional groups. It is of course important to remember that alcohol consumption among social class I (professional) groups is high. There is also some evidence that doctors have slightly elevated mortality rates compared with the general population for alcohol-related cirrhosis. However, other professional groups also have elevated rates, particularly lawyers.

\section{Junior doctors}

There have been three recent studies of psychological morbidity in junior doctors (Firth-Cozens, 1987, 1995; Humphris et al, 1994; Grainger et al, 1995). In 1986, Firth-Cozens reported a study of psychological distress in 318 fourth-year medical students at three different medical schools. She subsequently followed-up these students when they were junior house officers (Firth-Cozens, 1987) and then later as junior hospital doctors and general practitioners (GPs) (Firth-Cozens, 1995). She used the GHQ-30 with a cut-off of threequarters and found the estimated prevalence of emotional disturbance among the young doctors was approximately $50 \%$. This cut-off is less stringent than the three-quarter cut-off used on the GHQ-12 by Borrill et al (1996) who found an estimated prevalence of $32.4 \%$ for junior hospital doctors in their study.

Overwork was rated as being the most stressful factor (on average the doctors were working more than 90 hours per week) followed by talking to distressed relatives, serious treatment failures and effects on the doctors' personal lives (Box 2). FirthCozens found a high correlation between stressful aspects of work and GHQ scores. There were also high correlations between difficulties adjusting to changes in sleep patterns and depression. There was no correlation, however, between the number of hours worked on-call or the number of beds for which the doctors were responsible and levels of depression. Equally there was no relationship between alcohol intake and GHQ scores although
Box 2 Stress and job satisfaction

Job factors which junior doctors find most stressful

Overwork; talking to distressed relatives; serious treatment failures; and deleterious effects of work on their private life

Key causes of stress for GPs

Overwork; recent NHS changes and reforms; and patients' demands and expectations

Key components of job satisfaction in consultants

Good relationships with patients, relatives and staff; professional status; high level of autonomy; variety of work

Job satisfaction protects doctors in stressful jobs from psychological morbidity

one-fifth of doctors reported occasional or frequent bouts of heavy drinking.

Firth-Cozens (1987) found highly significant correlations between the levels of stress reported by junior doctors and their perception of their work. The more distressed the doctors were, the more negatively they saw aspects of their roles, particularly in relation to consultants. The most distressed doctors felt less able to discuss problems with senior colleagues and were less sure what was expected of them.

As with Borrill's findings, Firth-Cozens found a higher estimated prevalence of psychological morbidity in women doctors compared with men. This contrasted with her findings when the group were medical students as there was no difference at that time between the genders.

Grainger et al (1995) have recently reported a study of 235 pre-registration house officers in Birmingham. They used the Occupational Stress Indicator rather than the GHQ but found similar results to Firth-Cozens (1987) and Borrill et al (1996). They found high rates of psychological illness, with women reporting worse mental and physical health compared with men. Alcohol consumption was high, particularly for women doctors - twice as many women doctors were drinking in excess of the recommended limits for alcohol consumption compared with women in the general population. 
Nearly two-thirds of their sample regretted studying medicine, the main reasons cited for this being long hours of work, too much responsibility, and interference with the rest of their lives. More women than men experienced regret. Eighteen per cent of the house officers were unsure about continuing in medicine beyond senior house officer level and 20 had taken steps to leave the medical profession.

\section{Hospital consultants}

The most comprehensive study of psychological morbidity in hospital consultants is that by Ramirez et al (1996). They screened 1133 consultants in four different specialities: gastroenterology, surgery, radiology and oncology. They used the GHQ-12 with the same cut-off as Borrill et al (1996). The response rate was $78 \%$ and the overall estimated psychological morbidity for the group was $27 \%$. There was no significant difference between the different specialities. However, only $12 \%$ of the sample were women, reflecting the gender disparity in consultant appointments in the specialities studied.

When gender is taken into account, the figures from this study are very similar to those of Borrill et al, and also to the $23 \%$ prevalence of depression and anxiety (using the Hospital Depression and Anxiety Scale) found by Caplan (1994) in his sample of 65 consultants based in North Lincolnshire.

Of particular interest in this study (Ramirez et $a l, 1996)$ is the relationship that the researchers found between job stress, satisfaction and mental health. Doctors who reported high levels of job stress also reported high levels of burn-out (emotional exhaustion and high depersonalisation) and psychiatric morbidity. Job satisfaction, however, appeared to protect or buffer doctors against the negative relationship between job stress and burn-out and psychiatric morbidity. Doctors who reported high stress and low satisfaction were six times more likely to be probable cases on the GHQ than doctors who reported high stress but high satisfaction (see Box 2). Key components of job satisfaction for the doctors in this study were good relationships with patients, relatives and staff; professional status and self-esteem; high level of autonomy; and variety of work (see Box 2). Surgeons reported the highest levels of job satisfaction.

Consultants who felt insufficiently trained in communication skills and management skills reported high levels of depersonalisation and low personal accomplishment.

\section{Individual specialities}

Some older studies have suggested that particular specialities within medicine may be linked to especially high rates of psychiatric disorder. These have included psychiatry and anaesthetics. A recent unpublished study by the authors of over 100 psychiatrists in Manchester found very similar estimated prevalence rates of psychological morbidity to the other studies quoted above, suggesting that psychiatrists do not have higher rates of psychiatric disorder than other doctors. However, rates for suicide among psychiatrists, pathologists and anaesthetists have been reported as being higher than in some other medical specialities, although all the research in this area is rather dated.

Ramirez et al (1996) found that although there was no difference in psychological morbidity among the different consultant groups, radiologists reported a significantly lower sense of personal satisfaction on the MBI than gastroenterologists, surgeons or oncologists.

\section{General practice and community-based work}

The largest study of mental health in GPs (Cooper et al, 1989) surveyed a sample of 4000 GPs and received $1817(45.4 \%)$ replies. In contrast to other studies, the researchers found that women GPs had lower anxiety and depression scores than standardised population norms (Crown-Crisp Experiential Index) whereas male GPs had higher anxiety scores than the normal population but similar scores for depression. In a second, smaller study ( $n=917)$, four years later (Sutherland \& Cooper, 1993), and after the introduction of a new contract of employment for GPs, the researchers found a similar pattern of results. Women GPs had lower anxiety scores than population norms while male GPs still reported higher scores than population norms (Occupational Stress Index). Overall, GPs reported lower job satisfaction than in the previous survey, prior to the contract change. Job dissatisfaction was most strongly related to 'demands of the job and increased patient expectations'. These included increasing fear of assault, worry about complaints from patients, and adverse publicity from the media. Other key factors included the conflict between clinical care and new role demands, fear of making mistakes, 
mundane administrative work and insufficient resources. GPs reported that they no longer felt in control of the events that affected their ways of working.

Caplan (1994) surveyed 322 GPs, 81 hospital consultants and 121 senior hospital managers. He used the GHQ-30 and the Hospital Depression and Anxiety Scale (HAD). Scores on the HAD anxiety scale were similar among the three groups with approximately $30 \%$ scoring $\geq 10$. GPs, however, had significantly higher scores for depression than hospital managers, with consultants scoring between these two groups. GPs were also significantly more likely to report experiencing suicidal ideas than hospital consultants. This is in contrast to Borrill et al (1996) who found that NHS managers reported the highest rates of estimated psychiatric morbidity. The difference in the two studies may be due to differences in sampling and use of questionnaires, but it is also possible that in the past two years NHS managers have become more stressed. Caplan did not provide separate results for men and women, so the influence of gender on the overall scores cannot be assessed.

In a recent study examining stress in 532 GPs, 506 hospital doctors and 241 management consultants and accountants, McKevitt et al (1995) found that $72 \%$ of GPs and $50 \%$ of hospital doctors reported that their jobs were stressful most or all of the time. Both GPs and hospital doctors reported overwork as being a major cause of stress but GPs ranked 'other people's demands upon them and expectations upon them' much higher than hospital doctors, and also ranked 'changes in the way they were expected to do their job' much more highly than their hospital counterparts. This suggests the recent NHS reforms may be having a greater impact upon GPs than hospital doctors, although this may well change with time.

\section{Summary}

Most of the work looking at psychological morbidity and stress in the medical profession must be treated with caution as it is predominantly based upon the use of self-report questionnaires. Several recent large-scale studies have found remarkably consistent results, which suggest that doctors of all grades and specialities have higher rates of psychological morbidity than the general population. Rates of psychological morbidity are also high in other health service workers, particularly senior managers. Female hospital doctors have higher rates of psychological morbidity than their male counterparts, and also higher rates of psychological morbidity than other health service workers with the exception of senior managers. The situation appears to be somewhat different in general practice with less evidence of psychological distress in women GPs. The studies in general practice, however, have used different measures than the hospital-based studies, so the findings are not directly comparable. It is also the case that many women GPs work on a part-time basis, whereas part-time posts in the general hospital setting are still relatively rare.

In the past few years there are few senior doctors and GPs who have not experienced an increase in job demands and stress as a result of the health service reforms. Job satisfaction appears to protect doctors against the noxious effects of stress upon psychological ill health. In busy jobs, however, it is often the most enjoyable components of the job (e.g. teaching, running a special clinic or research) which are sacrificed in order to meet additional clinical or administrative demands. Strategies designed to reduce psychological morbidity in doctors will not only have to address individual coping and managerial skills, but also administrative issues relating to the increasing work loads of doctors and increasing patient expectations.

\section{References}

British Medical Association (1992) Stress and the Medical Profession. London: BMA.

- (1993) The Morbidity and Mortality of the Medical Profession. London: BMA Board of Science and Education.

Borrill, C. S., Wall, M.A., West, G. E., et al (1996) Mental Health of the Workforce in NHS Trusts. Final Report. Leeds: Institute of Work Psychology, University of Sheffield and Department of Psychology, University of Leeds.

Brooke, D, Edwards, G. \& Taylor, C. (1991) Addiction as an occupational hazard: 144 doctors with drug and alcohol problems. British Journal of Addiction, 86, 1011-1016.

Caplan, R. P. (1994) Stress, anxiety, and depression in hospital consultants, general practitoners, and senior health service managers. British Medical Journal, 309, 1261-1263.

Clare, A. W. (1990) The alcohol problem in universities and the professions. Alcohol and Alcoholism, 25, 277-285.

Cooper, C. L., Sloan, S. J. \& Williams, S. (1988) Occupational Stress Indicator Guide. Windsor: NFER-Nelson.

-_-, Rout, U. \& Faragher, B. (1989) Mental health, job satisfaction, and job stress among general practitioners. British Medical Journal, 298, 366-370.

Crown, S. \& Crisp, A. H. (1979) Manual of the Crown-Crisp Experiential Index. London: Hodder and Stoughton.

Edelwich, J. \& Brodsky, A. (1980) Burn-out: Stages of Disillusionment in the Helping Professions. New York: Human Sciences Press.

Firth-Cozens, J. (1987) Emotional distress in junior house officers. British Medical Journal, 295, 533-536.

- (1995) Stress in Doctors: A Longitudinal Study. Leeds: University of Leeds.

Firth, J. \& Morrison, L. (1986) What stresses health professionals? A coding system for their answers. British Journal of Clinical Psychology, 25, 309-310. 
Freundenberger, H. J. \& Richelson, G. (1980) Burnout: The High Cost of High Achievement. Garden City, NY: Doubleday.

Glass, D. G. \& McKnight, J. D. (1996) Perceived control, depressive symptomatology, and professional burnout: a review of the evidence. Psychology and Health, 11, 23-48.

Goldberg, D. \& Williams, P. A. (1988) A User's Guide to the General Health Questionnaire. Berkshire: NFER-Nelson.

Graham, J. R. (1987) The MMPI. A Practical Guide, 2nd edn. New York: Oxford University Press.

Grainger, C., Harris, E., Temple, J., et al (1995) Job satisfaction and health of house offices in the West Midlands. Health Trends, 27, 27-30.

Guthrie, E. A., Black, D., Shaw, C. M., et al (1995) Embarking upon a medical career: psychological morbidity in first year medical students. Medical Education, 29, 337-341.

Humphris, G., Kaney, S., Broomfield, D., et al (1994) Stress in Junior Medical and Dental Staff. Liverpool: University of Liverpool and Mersey Regional Health Authority.

Kelly, S., Charlton, J. \& Jenkins, R. (1995) Suicide deaths in England and Wales, 1982-1992: the contribution of occupation and geography. Population Trends, 80, 16-25.

Maslach, C. \& Jackson, S. (1986) Maslach Burnout Inventory (2nd edn). Palo Alto, CA: Consulting Psychologist's Press.

Miller, P. McC. \& Surtees, P.G. (1991) Psychological symptoms and their course in first-year medical students as assessed by the interval general health questionnaire (I-GHQ). British Journal of Psychiatry, 159, 199-207.

McCranie, E. W. \& Brandsma, J. M. (1988) Personality antecedents of burnout among middle-aged physicians. Behavioural Medicine, 14, 30-36.

McKevitt, C., Morgan, M., Simpson, J., et al (1995) Doctors' Health and Needs for Services. London: Nuffield Provincial Hospitals Trust.

Murray, R. M. (1976) Alcoholism amongst male doctors in Scotland. Lancet, ii, 729-731.

Pines, A., Aronson, E, \& Kafry, D. (1981) Burnout: From Tedium to Personal Growth. New York: Free Press.

Ramirez, A., Graham, J., Richards, M. A., et al (1996) Mental health of hospital consultants: the effects of stress and satisfaction at work. Lancet, 347, 724-728.

Richings, J. C., Khara, G. S., \& McDowell, M. (1986) Suicide in young doctors. British Journal of Psychiatry, 149, 475-478.

Steppacher, R. C. \& Mausner, J. S. (1974) Suicide in male and female physicians. Journal of the American Medical Association, 228, 323-328.

Sutherland, V. J. \& Cooper, C. L. (1992) Job stress, satisfaction, and mental health among general practitioners before and after introduction of new contract. British Medical Journal, 304, 1545-1548.

Zigmond, A. S. \& Snaith, R. P. (1983) The hospital anxiety and depression scale. Acta Psychiatrica Scandinavica, 67, 361-370.

\section{Multiple choice questions}

1. In doctors:

a the suicide rate is higher for men than women

b the prevalence of alcohol problems is higher than for all other professions

c job satisfaction protects those in stressful jobs from psychological morbidity

d high levels of job stress are associated with high levels of burn-out

e the prevalence of psychological morbidity is higher than in all other professional groups in the NHS.
2. In junior doctors:

a severity of depression is directly related to the amount of hours that they work

b over one-half are cases on the GHQ

c overwork is the most frequent cause of stress

d stress at work is highly correlated with GHQ scores

e the prevalence of psychological morbidity is higher in men than women.

3. In hospital consultants:

a surgeons report higher stress than all other groups

$b$ radiologists are the most satisfied with their jobs

c insufficient training in communication skills is associated with feelings of depersonalisation and low personal accomplishment

d a key component of job satisfaction is having a good relationship with patients

e approximately one-quarter score as cases on the GHQ.

4. In general practitioners:

a men report higher levels of anxiety than population norms

b women report higher levels of anxiety than population norms

c job dissatisfaction is strongly related to increasing patient expectations

d job satisfaction is currently lower than it was before the recent NHS changes

e overwork is a major cause of stress.

\begin{tabular}{|cclll|}
\hline \multicolumn{2}{|c|}{ MCQ answers } & & & \\
1 & 2 & 3 & 4 & \\
a F & a F & a F & a T \\
b F & b F & b F & b F \\
c T & c T & c T & c T \\
d T & d T & d T & d T \\
e F & e F & e T & e T \\
\hline
\end{tabular}

